eine Quelle reichen Gewinns, so daß der Erlös allein aus dem Flugstaub, den man für 1893 anf $273000 \mathrm{M}$ einschätrte, die aufgewandten Kosten gut verzinste. Wenn auch immer noch ein kleiner Anteil des Flugstaubes sich der Kondensation entzieht, so kann doch die Flugstaubfrage auf den Hüttenwerken, sowohl in hygienischer wie in wirtschaftlicher Beziehung, als gelöst gelten.

Weit schwieriger ist die Kondensation des schlimmsten Pflanzengiftes, der schwefligen Säure. Wasserrieseltürme, welche bei Salzsäure so erfolgreich sind, versagen hier den Dienst, weil die schweflige Säure im Wasser zu wenig löslich und in den Rauchgasen $z u$ stark verdünnt ist. $\mathrm{Cl} . \mathrm{W}$ j. $\mathrm{n} \mathrm{k}$ l e $\mathrm{r}$ fand in dem wasserberieselten Kalkstein ein Mittel, die $\mathrm{SO}_{2}$-Gase kleinerer Ultramarinfabriken zu entsäuren, aber für die gewaltigen Riuchmassen eines Hüttenwerks scheitert auch diese Rieselmethode schon an der Unmöglichkeit, die erforderlichen groken Wassermassen zu beschaffen. Der einzig gangbare Weg ist, die schweflige Säure konzentriert zu entwickeln und dann in Schwefelsäure zu verwandeln; diesen Weg haben die Freiberger Hütten seit 1857 beschritten; dasselbe haben die Hüttenwerke des Harzes, in. Stolberg und in Oberschlesien getan; überall sind neue Schwefelsäurefabriken entstanden, welche die Rauchschäden wesentlich vermindert und zugleich die Schwefelsäure zu einem leicht zugänglichen Industrieerzeugnis gemacht haben. Die Folge davon ist namentlich ein erfreuliches Auflolühen der Kunstdüngerfabriken gewesen, welche in Deutschland etwa die Hälfte der Schwefelsäure verbrauchen und in Superphosphat und Ammoniumsulfat umsetzen; und wir dürfen hier wohl der Kunst des Chemikers einige Anerkennung zollen, die es erreicht hat, aus der die Vegetation verwüstenden schwefligen Säure Stoffe herzustellen, mit deren Hilfe der Landwirt den Ertrag der Felder an Korn und Rüben auf das Doppelte und Dreifache gesteigert hat.

Dies Lob muß aber gleich etwas eingeschränkt werden. Bei vielen Hüttenprozessen, namentlich bei Verhüttung von Bleierzen, entweicht die schweflige Säure bisher in so verdünntem Zustande, daß man daraus in den Bleilsammern keine Schwefelsäure machen kann. Von cram Schwefel der Freiberger Erze werden nur $60-70 \%$ zugute gemacht, auf anderen Hüttenwerken noch weniger. Die große Halsbrücker Esse wirft mit ihren $10 \mathrm{cbm}$ Rauchgasen in 1 Sek. noch immer 201 (oder in 1 Tage 1700 cbm) reines Schwefligsäuregas aus, in einer Konzentration von 1:500; und wenn sich auch die früher verödeten Gelände in der Nähe der Hütte mit frischem Grün bedeckt haben, so macht sich heute in weiter Ferne, nach Osten bis $10 \mathrm{~km}$ weit, an den Fichtenbeständen des Tharandter Waldes eine wenn auch schwache Rauchwirkung bemerkbar, die man hinnehmen muß als das kleinere Übel, wenn man die Hütten nicht stillegen will.

Wix dürfen aber hoffen, das noch unvollständig gelöste Problem schließlich vollständig zu lösen. Auf den Hüttenwerken weht ein frischer Wind; der Bleiglanz kann heute nach dem neuen $\mathrm{H}$ e b e $\mathrm{r}$. lein schen Verfahren so vorteilhaft abgeröstet werden, daß die Friedrichshütte zu Tarnowitz und die Freiberger Hütte jetzt für die Bleierzröstgase Schwefelsäurefabriken erbauen. Ein weiterer großer
Fortschritt ist die Erfindung des Schwefelsäurekontaktverfahrens, eine der bedeutsamsten Errungenschaften der chemischen Technik der letzten Jahrzehnte, die auch durch einen Kondensationszwang veranlaßt ist, nämlich durch die Forderung der Synthese des Indigofarbstoffs, die dabei entwickelte schweflige Säure wieder zu rauchender Schwefelsäure zu kondensieren. Dies Kontaktverfahren vermag schweflige Säure noch in starker Verdünnung in Schwefelsäure zu verwandeln, wo die Bleikammern machtlos sind; es ist berufen, die Röstgase der Hütten noch besser als bisher zu verdichten, und wird auf rheinischen, oberschlesischen und den königl. sächsischen Hütten für diesen Zweck bereits stark in Anspruch genommen.

Nur den gewöhnlichen Rauchgasen der Steinkohlenfeuerungen läßt sich auf diese Weise nicht beikommen, denn es kann unmöglich jedem Schornsteine eine Sohwefelsäurefabrik angeschlossen werden. Aber hier gibt es ein anderes sehr wirksames Mittel, nämlich Verdünnung bis zur Unschädlichkeitsgrenze. Die Mengen schweflige Säure und Schwefelsäure, welche die Städte Hannover und Linden aus ihren Steinkohlen in die Luft schicken, wenigstens $5000 \mathrm{cbm}$ Schwefligsäuregas täglich (bei einem sehr niedrig angenommenen Gehalte der Kohlen an $1 / 2 \%$ flüchtigem Schwefel) sind absolut genommen weit größer, als die von den beiden Freiberger oder den drei Oberharzer Hütten entwickelten Säuremengen, aber sie treten aus vielen über eine große Fläche verteilten Schornsteinen, und aus diesen in viєl größerer Verdünnung aus; und die Verdünnung ist entscheidend, andernfalls würde in unserer Stadt längst kein Blatt mehr grünen. Das unendliche Luftmeer, welches unsere Wohnstätten umgibt, vermag diesen Schädling, die Säuren des Kohlenrauchs, bei richtiger Behandlung leicht unschädlich zu machen. Gehen wir auf dem von der Natur vorgezeichneten Wege weiter, dezentralisieren wir unsere Industrie und unsere Städte, so wird der Sieg über die schädlichen Gase ein vollständiger werden. Und dieser Sieg ist mit Unterstützung der staatlichen und städtischen Behörden wohl gangbar, denn die Entwicklung unserer Großstädte drängt so wie so zur Dezentralisation; der Stadtbewohner verlangt mit Sehnsucht mehr Raum, Luft, Licht und mehr Natur.

\section{Bemerkungen zur Theorie der Molekularschwingungen.}

\section{Von Privatdozent Dr. G. HelLer, Leipzig.}

(Eingeg. den 22./7. 1907.)

Durch experimentelle Untersuchungen auf organischem Gebiete wurde ich vor drei Jahren veranlaßt, meine Anschauungen über die Schwingungen der Atome und Moleküle darzulegen (Liebigs Ann. 332, 286). Eine Reihe von Beobachtungen, welche in dieser Abhandlung angeführt sind, sowie theoretische Überlegungen führten mich zu der Annahme, daß die Moleküle im nicht reagierenden Zustande eine andere Schwingungsform besitzen, als in dem Momente, wenn sie in eine chemische Reaktion ein- 
troten. Für letzteren Zustand habe ich den Ausdruck ,Reaktionsschwingung" vorgeschlagen.

Diese Erörterungen sind anscheinend ziemlich unbeachtet geblieben. So mußte ich Herrn R a s $\mathrm{chig}$ auf meine Ausführungen aufmerksam machen, nachdem er in einer Abhandlung, betitelt „Gedanken über Katalyse" (diese Z. 19, 1748 [1906]) die Formveränderung der Moleküle als dasjenige bezeichnet hatte, was für das Zustandekommen der chemischen Reaktionen und speziell der katalytischen Erscheinungen von Wichtigkeit und ausschlag. gebend sei. Von anderer Seite wurde Herrn $\mathrm{R}$ a s $\mathrm{ch} \mathrm{ig} \mathrm{auch} \mathrm{vorgehalten,} \mathrm{daß} \mathrm{er} \mathrm{mit} \mathrm{seinen} \mathrm{Dar-}$ legungen in fremdes Arbeitsgebiet eingedrungen sei, und er sah sich dann veranlaßt, zu erklären: ,Bleibt also von allem, was ich vorschlug, als neu nur noch der Gedanke übrig, daß der veränderten Molekülform veränderte chemische Eigenschaften des Moleküls entsprechen."

Aber auch das ist, soweit das organische Gebiet in Betracht kommt, in meiner Abhandlung (für die ich natürlich auch nicht in allen Punkten Originalität beanspruche) schon ausgeführt, nämlich bei der Erklärung des Begriffes der Reaktionsschwingung und bei den dafür angeführten Beispielen, die gerade auf organischem Gebiete in einzelnen Fällen ziemlich prägnant sind.

Im übrigen sehe ich durchaus keinen Grund ein, Herrn Raschig irgend welche Vorwürfe über seine Exkursion $z u$ machen. Wenn auch in der Regel der Experimentator selbst seine Schlüsse aus dem Versuchsmaterial zieht und meist am besten beurteilen kann, nach welcher Richtung eine theoretische Erwägung neue Gesichtspunkte zutage fördern wird, so halte ich diese Art der Besprechung für vielleicht eben so berechtigt, wie die registrierende und klassifizierende Tätigkeit, wie sie in letzter Zeit mehr als je in der Abfassung kurzer Leitfäden auf dem Gebiete der allgemeinen und analytischen Chemie so ansgiebig geubt wird. In den Stunden, die nicht den auf experimenteller Grundlage beruhenden chemischen Studien gewidmet sind, wird man auch gern eine solche etwas leichtere Lektüre lesen und fast immer die eine oder andere Anregung erhalten.

Von Herrn W o h.l (Berl. Berichte 40, 2290) bin ich nicht richtig verstanden worden. Er ist der Ansicht, daß die bei einem chemischen Vorgange auftretende Wärme veränderte Reaktionen hervorbringen könne, denn er sagt : „Da bei langsam verlaufenden Vorgängen nicht alle Molekeln zugleich reagieren können, und da die Ableitung der Reaktionsenergie, d. i. die Verteilung der Wärmetönung, die in den reagierenden Molekeln auftritt, auf das ganze Medium Zeit erfordert, so ist unzweifelhaft, daß die reagierenden Moleküle und ihre unmittelbaren Umsetzungsprodukte für sehr kurze Zeit, nämlich bis die Fortleitung der Reaktionswärme erfolgt ist, in einem Zustande sind, der einer höheren Temperatur entspricht, als sie sonst Molekeln bei der vom Thermometer angezeigten Temperatur erreichen" (diese Z. 20, 1175 [1907]). Er fügt dann unter Erwähnung meiner Anschauungen hinzu, daß die einfache Beziehung der Reaktionsenergie zur Temperatur, die weitere besondere Voraussetzungen unnötig macht, bisher noch nicht in Betracht gezogen zu sein scheint.

Die Wohlsche Überlegung ist meines Er. achtens aber nicht ganz zutreffend. Die Wirkung der Wärme bei der Reaktion findet selbstverständlich statt, aber sie kommt erst in zweiter Linie in Betracht. Der primäre Vorgang ist offenbar folgender. Bei einem exothermischen Prozeß wird bekanntlich in die neu entstehende Verbindung nicht dieselbe Menge Energie mit hinübergenommen, welche in die Reaktion eingetreten ist. Die in den reagiorenden Moleküilez vorhandene Energiemenge, speziell aber die überschüssige Energie wird die Reaktionsschwingung veranlassen und die neugebildeten Moleküle, welche also sich in einem Zustande befinden, der ganz exzeptionell ist, müssen offenbar unter Umständen besondere Reaktionen zeigen. Wenn dann das Plus an Energie nach außen tritt, wandelt es sich in Wärme um; aber diese wirkt erst von außen her auf das Molekül ein und verteilt sich sofort, da sie auch die Nachbarmoleküle in Mitleidenzieht. Immerhin können ja lokale Überhitzungen stattfinden, aber dieselben dürften keineswegs erheblich sein; außerdem müßten ihre Wirkungen stets mit der einer späteren Temperaturerhöhung zusammenfallen. Die eigentliche Reaktionsschwingung in meinem. Sinne hat mit diesem Vorgange nichts mehr zu tun.

Im übrigen ist die Anschauung, daß bei Ein. tritt von Energicumschaltungen stets eine Umwandlung in Wärme stattfindet, durchaus nicht in allen Fïllen haltbar; so sind nach der allgemeinen Auffassung die Leuchterscheinungen in tierischen und pflanzlichen Organismen an chemische Vorgänge geknüpft; es findet also hier eine Umwandlung von chemischer Energie in Lichtschwingung statt. Nach noueren Untersuchungen von $\mathrm{T} \mathrm{ra} \mathrm{ut} z$ sind derartige mit Leuchterscheinungen verbundene Reaktionen recht häufig, und es ist nicht unwahrscheinlich, daB bei diesen Umformungen Energie noch in anderer, nicht meBbarer Weise austritt, so daß die Bestimmung der Wärme kein genaues Bild des Vorganges gibt.

Ich möchte zur Illustrierung meiner Anschauung nochmals auf die Tatsache hinweisen (Liebigs Ann. 324, 134), daß die Benzoylierung der Anthranilsäure in Pyridinlösung bei Temperaturen unter $0^{\circ}$ direkt zum Benzoylanthranil führt. Es bildet sich natürlich zunächst Benzoylanthranilsäure, welche dann unter dem Einfluß der Reaktionsschwingung sofort in Benzoylanthranil übergeht. Natürlich wirkt sekundär auch die bei der Umwandlung der Energie auftretende Wärme ein, aber da fertiggebildete Benzoylanthranilsäure selbst beim Siedepunkte der Pyridinlösung kein Wasser abspaltet, wie sich das bei dem basischen Lösungsmittel eigentlich von selbst versteht, so ist die sekundäre Wärmewirkung wohl ausgeschlossen, da innerhalb einer Flüssigkeit eine noch höhere lokale Uberhitzung wenig wahrscheinlich ist. Die Reaktionsschwingung ist also hier stärker als die Affinität der Benzoylanthranilsäure zum Pyridin; sie vermag die Salzbildung zu vermeiden und den Ringschluß unmittelbar herbeizuführen.

Ausgeschlossen erscheint die W o h l sche Auffassung auch durch die von $\mathrm{E}$ in $\mathrm{h}$ or $\mathrm{n}$ and seinen Schülern beobachtete Tatsache (Berl. Berichte 16, 2209, 3004; 1\%, 595, 2021), da 3 die verschiedenen isomerenNitrophenyl- $\beta$-brompropions äuren beim Behandeln mit kalter Sodalösung das Lacton der ent- 
sprechenden Nitrophenylmilchsäuren absscheiden, denn es ist bisher nicht gelungen, die freien Säuren wieder in das Lacton äberzuführen.

\section{Zur Kenntnis des Chlorkalks.}

(Erwiderung an Herrn E. Schwarz.)

$$
\text { Von Hugo Dir\%. }
$$

(Eingeg. den 1./8. 1907.)

Für die Leser dieser Zeitschrift ist es wohl nicht nötig, ausdrücklich festzustellen, daß es nicht ,der 'lon der Kritile und der Mangel an experimentellen Beweisen" ist, die Herru E. Se h wa $\mathrm{xz}^{1}$ ) „,jeder weiteren Polemik entheben“. Unter ,experimentellen Beweisen" versteht wohl Sohwarz

1) Diese Z. $\mathbf{7 0}, 1280$ (1907). beispielsweise die Art und Weise, wie e $\mathrm{r}^{2}$ ) die von ihm neu ,erfundene" Eigenschaft des Chlorkalks (daß dieser beim Frhitzen. kein Chlor ahgibt!) auch nicht durch e in Versuchsresultat zu begründen gesucht hatte, während er andererseits ,Mangel an experimentellen Beweisen " die Feststellung m e i $n$ ers $e$ it $s^{3}$ ) nennt, daß nach allen von mir $z a h$ lenmä $B$ ig angef ührten Versuchser. $\mathrm{g}$ e bn is se $\mathrm{n}$ einer Reihe von Forschern seine Behauptung unrichtig ist. Meine, wie ich glaube, streng sachliche Kritik seiner eigenartigen Ansichten hätte übrigens Herr $\mathrm{Sch}$ w a $\mathrm{r} z$ sich und mir vielleicht ersparen können, wenn er sich $v$ or der Veröffentlichung seiner Abhandlang etwas eingehender mit der Chlorkalkfrage beschäftigt hätte.

B r ï n n, Technische Hochschule im Juli 1907.

2) Dies? Z. 20, 138 (1907)

3) Diese Z. 20, 754 (1907).

\section{Referate.}

\section{4. Keramik, Glas, Zement, Bau- materialien.}

M. Simonis. Zur Verwendung von geschmolzener Magnesia, Quarzglas und Carborund als schwerschmelzbare Materialien. (Sprechsaal 40, 3 [1907]. Charlottenburg.)

Da die Magnesia bis zu ihrem Schmelzen fortwährenden Veränderungen unterliegt - Zunahme der Dichte von 3,19-3,65, Schwindung, Reißen -, so versagt bei 'Temperaturen zwischen Platinschmelze und Magnesiumschmelze (SK. 36-42 und höher) jedes niedriger, also etwa in Hauptporzellanglutbrand gebrannte Material, wenn größere Gegenstände daraus zu erhitzen sind. Die in einer früheren Veröffentlichung (Sprechsaal 39, 14, 15 [1906]) über elektrische Versuchsöfen angedeuteten Versuche, ein Material zu finden, das bei diesen Temperaturen sich zur Herstellung der Heizrohre eignet, haben dazu geführt, Gegenstände aus geschmolzener Magnesia herzustellen; mit Heizrohren aus diesem Material sind Vorgänge bei SK. 37-42 der direkten Beobachtung mit dem Auge zugänglich geworden. Als Heizrohr für klaren Brand bis etwa 1300 eignen sich Muffeln und Tiegel aus Quarzglas, die gasdicht sind, nicht springen und jetzt zu niedrigem Preis im Handel erhältlich sind. Als schwerschmelzbares Material, das in hervorragender Weise Temperaturschwankungen erträgt, eignet sich Carborund verschiedener Körnung, den man etwa durch Kaolin bindet.

Nn.

\section{P. Rohland. Die Erhärtung des Schwerspats.} (Sprechsaal 39, 1417 [1906].)

Verf. bespricht die Theorie des Härtens und berichtet über mehrere Versuche, die er hinsichtlich des Härtungsvorganges von Bariumsulfat mit einem Gemisch von Calciumcarbonat, Soda und Ton nebst Wasser angestellt hat. Das Verfahren war folgendes : Fein zerteilter Schwerspat wurde mit den oben erwähnten Substanzen zusammengerührt, zu Stei- nen geformt, getrocknet und zwischen $500-600^{\circ}$ etwa bei SK 022 gebrannt. Die quantitative Analyse der gehärteten Masse ergab:

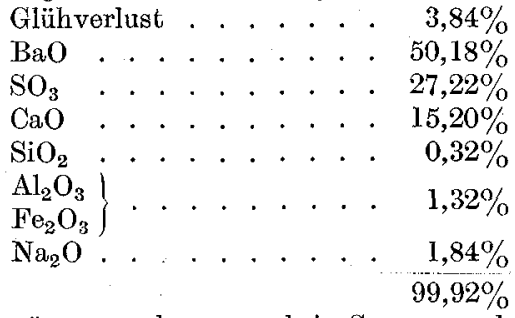

Kohlensäure war kaum noch in Spuren vorhanden, ein Beweis, daß Natriumcarbonat, das nach neueren Messungen erst bei $1045-1098^{\circ}$ dissoziiert, hier schon bei verhältnismäßig niedriger Temperatur $\left(500-600^{\circ}\right)$ gespalten warde. Die erhärtete Masse schmilzt vor dem Gebläse wie Glas und trübt sich beim Erkalten wieder. In konz. Schwefelsäure löst sie sich fast vollständig auf. Härte $=2,5$ der Skala (Härte des natürlichen Schwerspats $=3$ ). $N n$.

W. Krumbhaar. Der Gips. (Tonind.-Ztg. 30, 2173, 2207,2258 [1906].)

Eine chemisch-technische Studie über die Abarten des Calciumsulfats. $N n$.

P. Rohland. Uber das hydratisehe Wasser der Tone, Zemente und einiger anderer Stoffe. (Sprechsaal 40, 2 [1907]. Stuttgart.)

Durch Entfernung des Krystallwassers eines Salzes werden im allgemeinen keine chemischen Eigenschaftsänderungen hervorgerufen; solche treten erst ein, wenn das hydratische Wasser entweicht. An der Hand zahlreicher Beispiele von Materialien der Tonwarenindustrie und dieser nahestehender Produkte weist Verf. darauf hin, welche wichtige Rolle dem Hydratwasser zukommt. Mehr als die Temperaturdifferenzen, welche zwischen der Abgabe von Kry. stall-, Hydrat- oder Konstitutionswasser statthaben, fallen die physikalisch-chemischen Änderungen, die durch Abgabe des Hydratwassers hervorgerufen 